\title{
GJ 1214: Rotation period, starspots, and uncertainty on the optical slope of the transmission spectrum ${ }^{\star}, \star \star$
}

\author{
M. Mallonn ${ }^{1}$, E. Herrero ${ }^{2,3}$, I. G. Juvan ${ }^{4,5,6}$, C. von Essen ${ }^{7}$, A. Rosich ${ }^{2}$, I. Ribas ${ }^{2}$, T. Granzer ${ }^{1}$, \\ X. Alexoudi ${ }^{1}$, and K. G. Strassmeier ${ }^{1}$ \\ ${ }^{1}$ Leibniz-Institut für Astrophysik Potsdam, An der Sternwarte 16, 14482 Potsdam, Germany \\ ${ }^{2}$ Institut de Ciéncies de l'Espai (ICE, CSIC), Campus UAB, Carrer de Can Magrans sn, 08193 Cerdanyola del Vallès, Spain \\ ${ }^{3}$ Institut d'Estudis Espacials de Catalunya (IEEC), C/Gran Capitá 2-4, Edif. Nexus, 08034 Barcelona, Spain \\ ${ }^{4}$ Space Research Institute, Austrian Academy of Sciences, Schmiedlstrasse 6, 8042, Graz, Austria \\ ${ }^{5}$ Institut für Geophysik, Astrophysik und Meteorologie, Karl-Franzens-Universität, Universitätsplatz 5, 8010 Graz, Austria \\ ${ }^{6}$ Institut für Astro- und Teilchenphysik, Universität Innsbruck, Technikerstrasse 25, 6020 Innsbruck, Austria \\ 7 Stellar Astrophysics Centre, Department of Physics and Astronomy, Aarhus University, Ny Munkegade 120, 8000 Aarhus C,
}

e-mail: mmallonn@aip.de Denmark

Received 15 November 2017 / Accepted 6 March 2018

\begin{abstract}
Aims. Brightness inhomogeneities in the stellar photosphere (dark spots or bright regions) affect the measurements of the planetary transmission spectrum. To investigate the star spots of the M dwarf GJ 1214, we conducted a multicolor photometric monitoring from 2012 to 2016.

Methods. The time-series photometry was analyzed with the light curve inversion tool StarSim. Using the derived stellar surface properties from the light curve inversion, we modeled the impact of the star spots when unocculted by the transiting planet. We compared the photometric variability of GJ 1214 to published results of mid- to late M dwarfs from the MEarth sample.

Results. The measured variability shows a periodicity of $125 \pm 5$ days, which we interpret as the signature of the stellar rotation period. This value overrules previous suggestions of a significantly shorter stellar rotation period. A light curve inversion of the monitoring data yields an estimation of the flux dimming of a permanent spot filling factor not contributing to the photometric variability, a temperature contrast of the spots of $\sim 370 \mathrm{~K}$ and persistent active longitudes. The derived surface maps over all five seasons were used to estimate the influence of the star spots on the transmission spectrum of the planet from 400 to $2000 \mathrm{~nm}$. The monitoring data presented here do not support a recent interpretation of a measured transmission spectrum of GJ 1214b as to be caused by bright regions in the stellar photosphere. Instead, we list arguments as to why the effect of dark spots likely dominated over bright regions in the period of our monitoring. Furthermore, our photometry proves an increase in variability over at least four years, indicative for a cyclic activity behavior. The age of GJ 1214 is likely between 6 and 10 Gyr.

Conclusions. The long-term photometry allows for a correction of unocculted spots. For an active star such as GJ 1214, there remains a degeneracy between occulted spots and the transit parameters used to build the transmission spectrum. This degeneracy can only be broken by high-precision transit photometry resolving the spot crossing signature in the transit light curve.
\end{abstract}

Key words. techniques: photometric - stars: activity - stars: individual: GJ1214 - starspots

\section{Introduction}

For the vast majority of currently known exoplanets, direct spectroscopy is hampered by the strong brightness contrast between the host star and the planet, and by their small angular separation. However, transiting extrasolar planets offer the opportunity to perform indirect spectroscopy of these planets. Planetary spectroscopy using the transit event, the so-called transmission spectroscopy, searches for spectral modifications of the part of the star light shining through the planetary atmosphere during transit. The opacity of the planetary atmosphere is a function of wavelength, dependent on chemical composition (Fortney et al. 2010). Relative differences in opacity can be measured

\footnotetext{
${ }^{\star}$ Based on data obtained with the STELLA robotic telescopes in Tenerife, an AIP facility jointly operated by AIP and IAC.

${ }^{\star \star}$ The photometry tables are only available at the CDS via anonymous ftp to cdsarc.u-strasbg.fr (130.79.128.5) or via http://cdsarc.u-strasbg.fr/viz-bin/qcat?J/A+A/614/A35
}

by comparing photometric transit light curves taken at multiple wavelengths (Bean et al. 2010; Pont et al. 2013; Kreidberg et al. 2014; Lendl et al. 2016; von Essen et al. 2017; Kirk et al. 2017). At high opacity, the planetary radius appears larger than at wavelengths of weaker opacity (Sing et al. 2016; Sedaghati et al. 2017; Mallonn \& Wakeford 2017).

However, brightness inhomogeneities caused by magnetic activity, starspots or faculae (Strassmeier 2009), modify the derived transit parameters and can mimic spectral features of the planetary atmosphere (e.g., Oshagh et al. 2013b; Barstow et al. 2015; Herrero et al. 2016). For example, a trend of increasing planetary radius toward blue wavelengths can be caused by scattering properties in the atmosphere of the planet, but also by the presence of starspots on the visible hemisphere of the host star (Pont et al. 2013; McCullough et al. 2014). One exoplanet that received a lot of attention in recent years is the super-Earth GJ 1214b (Charbonneau et al. 2009). Its mean density can be explained by different bulk compositions. The possible scenarios 
can be distinguished by spectroscopy of the planetary atmosphere (Miller-Ricci \& Fortney 2010). Because of the relatively large transit depth, such measurements were feasible and conducted by different groups from space (e.g., Berta et al. 2012a; Kreidberg et al. 2014) and from the ground (e.g., Bean et al. 2010; Croll et al. 2011; de Mooij et al. 2013; Nascimbeni et al. 2015). The planet spectrum was found to be flat from blue optical wavelength to the near-infrared (NIR).

The host star GJ 1214 is an active mid M dwarf of 0.176 solar masses (Anglada-Escudé et al. 2013) that shows photometric variability due to active regions rotating in and out of view (Charbonneau et al. 2009). However, no clear picture of its activity pattern could be derived so far. Several teams performed photometric monitoring campaigns (e.g., Berta et al. 2011; Nascimbeni et al. 2015), but drew different conclusions. Tentative detections of a periodicity in the variability (which is linked to the stellar rotation period) ranges from $\sim 40$ days (Narita et al. 2013) to $\sim 80$ days (Nascimbeni et al. 2015). Newton et al. (2016a) still lists the stellar rotation period as unknown. The measured amplitude of variability ranges from below $2 \mathrm{mmag}$ in Cousin $I$ (Gillon et al. 2014) up to $3 \%$ in the Sloan $g$ band (Teske et al. 2013). In principle, this range of results could be partly explained by an insufficiently long monitoring of the host star, and by an unknown evolution of the activity patterns on the host star.

The photometric variability so far was always interpreted as dark spots rotating in and out of view. Starspots on GJ 1214 have been seen in transit photometry as bumps in several transit light curves (Berta et al. 2011; Carter et al. 2011; Bean et al. 2011; Narita et al. 2013; Nascimbeni et al. 2015). A different interpretation of GJ 1214's variability was recently given by Rackham et al. (2017), who measured an offset toward lower values in the planetary transmission spectrum of the optical data compared to previously published NIR results. They interpreted it as caused by bright regions in the stellar photosphere.

In this work, we want to address the stellar activity and its influence on the transmission spectrum through the analysis of five seasons of multicolor photometric monitoring. GJ 1214 was observed as part of our monitoring survey VAriability MOnitoring of exoplanet host Stars (VAMOS, Mallonn et al. in prep.) from 2012 to 2016 in the Johnson/Cousins filters $B, V$, and $I$. Section 2 describes the observations and the data reduction. In Sect. 3, we derive the results for the photometric variability in general and the results obtained by the light curve inversion done with StarSim (Herrero et al. 2016). In Sect. 4, we compare the variability of GJ 1214 to other mid- to late M dwarfs of the MEarth sample, we discuss the difficulty to extract the optical slope of the planetary transmission spectrum in case of an active host star, and give a discussion on the cyclic activity behavior and the age of GJ 1214. We finish in Sect. 5 with a conclusion.

\section{Observation and data reduction}

The exoplanet host star GJ 1214 was monitored photometrically over five observing seasons with the robotic telescope STELLA (Strassmeier et al. 2004), located on Tenerife, and its wide-field imager WiFSIP (Granzer et al. 2010; Weber et al. 2012). The duration of observations per season and number of acquired images is summarized in Table 1. The data of 2012, and partly of 2013, have already been analyzed and published in Nascimbeni et al. (2015). They are also included here for an overall analysis. A malfunction of the CCD cooling in the first data points of 2012 was unnoticed in Nascimbeni et al. (2015). These points are excluded here.
WiFSIP offers a field of view (FoV) of $22^{\prime} \times 22^{\prime}$ on a scale of $0.32^{\prime \prime}$ pixel $^{-1}$. The detector is a single $4096 \times 4096$ back-illuminated thinned CCD with $15 \mu \mathrm{m}$ pixels. In 2012 , the observations were performed in blocks of five exposures in Johnson $V$ ( $150 \mathrm{~s}$ exposure time) and five exposures in Cousin $I(60 \mathrm{~s})$. In 2013, we used Johnson $B$ instead of $I$ because of its higher sensitivity to starspots and observed in blocks of three exposures per filter with $300 \mathrm{~s}(B)$ and $150 \mathrm{~s}(V)$ exposure time. In 2014 to 2016, the observing blocks consisted of two exposures in $B$ $(400 \mathrm{~s})$ and two in $V(200 \mathrm{~s})$.

The data reduction was done with the same ESO-MIDAS routines already used for similar monitoring programs of exoplanet host stars with STELLA WiFSIP (e.g., Mallonn et al. 2015a,b). Aperture photometry was executed with the publicly available software SExtractor (Bertin \& Arnouts 1996). We employed the SExtractor aperture option MAG_AUTO, which is an elliptical aperture adjusted individually per frame according to the object light distribution. This option accounts for the changing observing conditions from night to night over the duration of the campaign. For further detail of the data reduction we refer to Mallonn et al. (2015b) and Nascimbeni et al. (2015). As comparison stars we used the same four stars within $3^{\prime}$ of GJ 1214 as in Nascimbeni et al. (2015). We verified that the variability pattern in the derived light curve is not significantly influenced by the choice of the comparison stars. We discarded data of lower quality by applying stringent selection criteria which are airmass higher than 1.5 , flux less than $50 \%$ of average, and unusual values of sky background, width and elongation of point-spread-function, and CCD temperature. From 2013, the criterion of airmass $X<1.5$ was programmed in the STELLA telescope software that triggers the observations. Therefore, a higher fraction of the observed images met the selection criteria. The data points in 2012 and 2013 were binned per observing block. The resulting light curve is shown in Fig. 1.

\section{Results}

\subsection{Stellar variability}

Within the time span of our monitoring, the years 2012 to 2016, there were two other variability measurements of the host star published. Teske et al. (2013) observed multiple transits in 2012 and compared the out-of-transit magnitudes of GJ 1214 taken in Sloan $g$ band with STELLA WiFSIP in their Fig. 4. The variability pattern in these data agrees well with our measurements from 2012 in the fact that the star was at a similar brightness in late May and late August 2012, while it was dimmer in June. The monitoring of Narita et al. (2013) overlaps the monitoring presented here only by a few days. However, in their Fig. 6, Narita et al. (2013) extrapolated the measured variability pattern to earlier dates. This extrapolation contradicts our measurements as it states a brightness minimum around JD 2456120 for which we measure a maximum in this work.

We computed a Lomb-scargle periodogram for each season individually and for combined seasons. Here, we merged the data sets of the available colors. The four seasons 2013-2016 $B+V$ consistently showed a signal $P>100$ days, whereas the periodogram of season 2012 reaches peak power at $P \sim 70$ days as already shown in Nascimbeni et al. (2015). We obtain the most significant signal in the periodogram for the data $B+V$ 20142016 at a period of $125 \pm 5$ days (Fig. 2). The uncertainty is the HWHM of the peak in the periodogram.

Our STELLA $B$ and $V$ band data measure a full amplitude variability of $5.3 \%$ in $V$ and $B$, which is the highest variability 
Table 1. STELLA monitoring observations of GJ 1214.

\begin{tabular}{lccccc}
\hline \hline Year & 2012 & 2013 & 2014 & 2015 & 2016 \\
\hline Time range & Mar 21-Oct 21 & Mar 24-Oct 4 & Feb 13-Oct 16 & Feb 4-Oct 11 & Feb 6-Sep 3 \\
Indiv. nights & 110 & 81 & 116 & 50 & 53 \\
Filter & $V / I$ & $B / V$ & $B / V$ & $B / V$ & $B / V$ \\
$\mathrm{n}_{\text {data }}$ & $57 / 72$ & $43 / 47$ & $206 / 206$ & $68 / 76$ & $73 / 77$ \\
\hline
\end{tabular}

Notes. The data of 2012 and part of 2013 are presented in Nascimbeni et al. (2015). The number of data points $n_{\text {data }}$ denotes the value after removal of low-quality data and in 2012 and 2013 after binning the data per observing block.

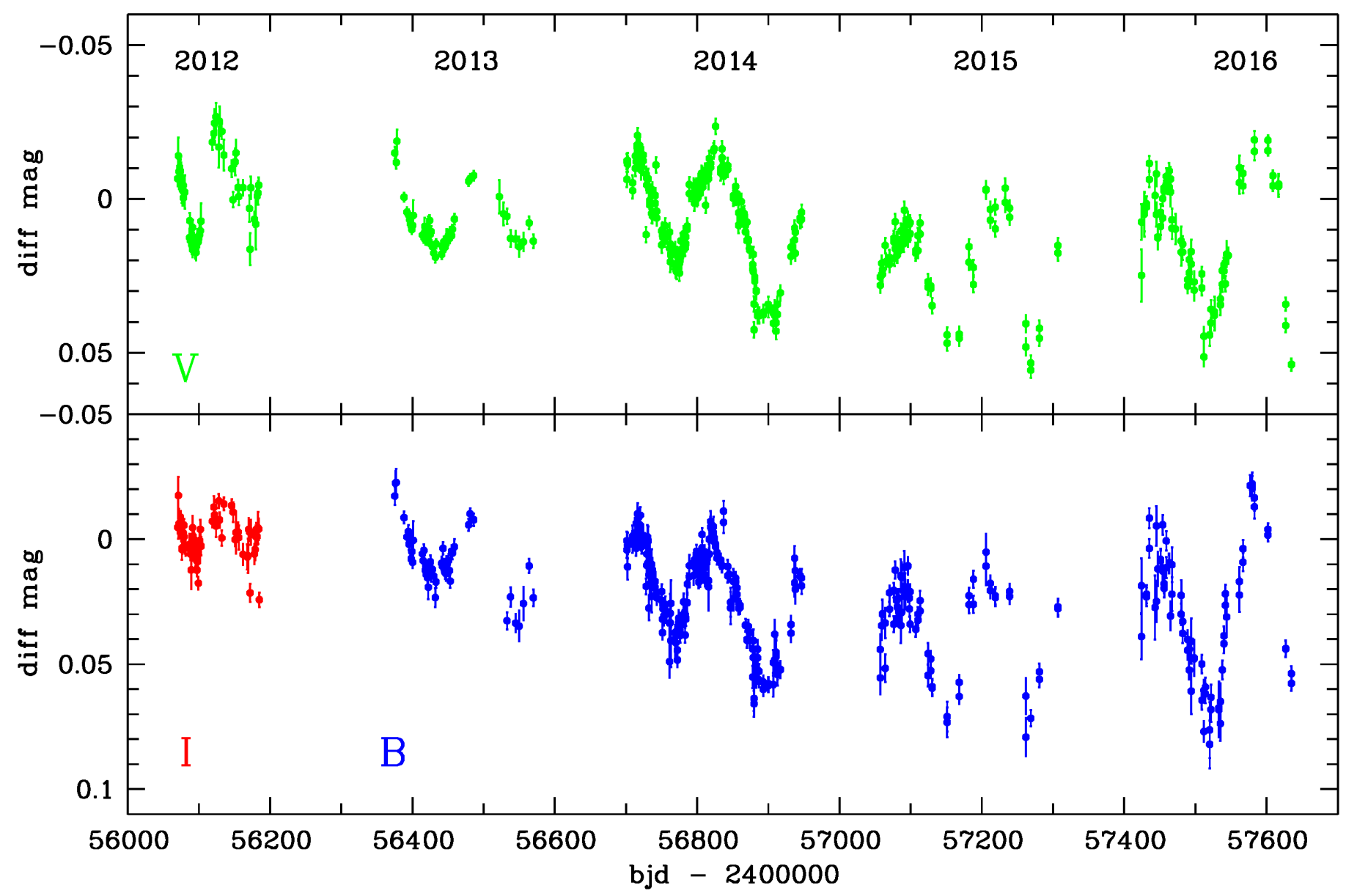

Fig. 1. Differential light curve of GJ 1214 covering the time span from 2012 to 2016 . Blue data points denote measurements in the Johnson $B$ filter, whereas green and red points indicate observations in the Johnson $V$ and Cousin $I$ filter, respectively.

measured so far for this exoplanet host star. Other papers containing long-term photometry of GJ 1214 from 2010 to 2012 already showed a rise in variability amplitude. In 2010, Berta et al. (2011) measured a full-amplitude in the $V$ band of $1.3 \%$. Nascimbeni et al. (2015) reported an increase in amplitude from their analysis of the 2012 STELLA data to about $2 \%$ in $I$. For the same year 2012, Teske et al. (2013) obtained an out-of-transit variability of about $3 \%$ in the bluer Sloan $g$ band. We discuss the increase in amplitude of photometric variability from the year 2008 to 2016 as indicators for a stellar activity cycle in Sect. 4.2 .

\subsection{Spot modeling with StarSim}

The host star GJ 1214 is known to present stellar variability due to inhomogeneities in the photosphere (Berta et al. 2011;
Nascimbeni et al. 2015). Time series multicolor photometry allows for the determination of the amount and longitude distribution of active regions on the stellar surface. From this, it is also possible to derive the temperature contrast of apparent starspots in the photosphere (Strassmeier 2009).

Among the different spot modeling techniques, we chose the StarSim tool (Herrero et al. 2016) to analyze the data described in Sect. 2. The methodology consists of the surface integration of fluxes using Phoenix BT-Settl atmosphere models (Allard et al. 2011). Different temperatures were considered for the spots and for the quiet photosphere. The total flux produced by the visible stellar surface was obtained by adding the contribution of the visible surface elements, considering the limb darkening computed from Kurucz ATLAS9 models (Castelli \& Kurucz 2004). StarSim models the surface of an active star by considering a certain amount of cool spot elements. The number of active 


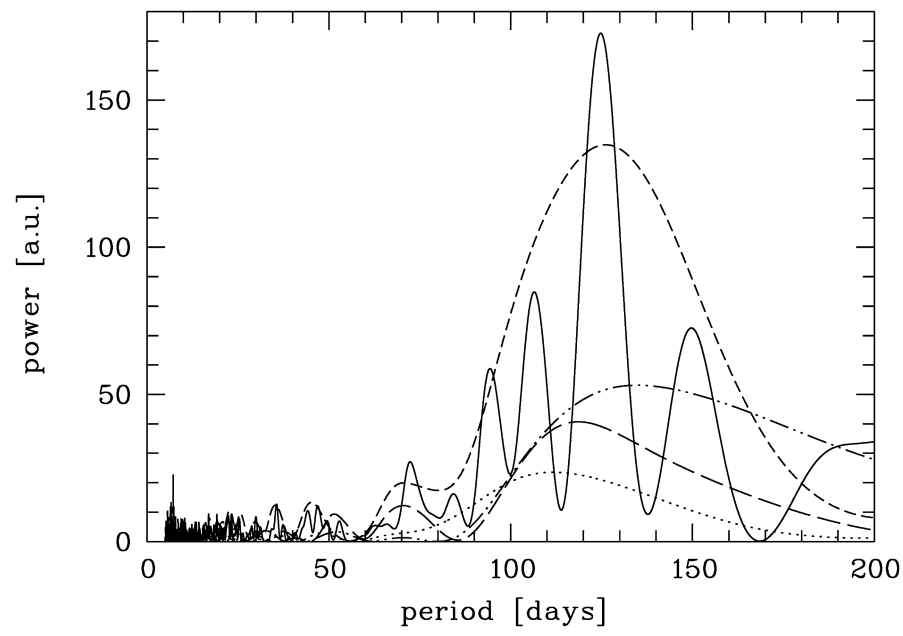

Fig. 2. Lomb-Scargle periodogram of the differential light curve of GJ 1214. The black line gives the combined data set $B+V$ from 2014 to 2016, the dotted, short dashed, long dashed, and dashed-dotted lines shows the result for the $B+V$ data of individual years 2013, 2014, 2015, and 2016, respectively.

surface elements, as well as their locations and lifetimes, are free parameters of the model. This number corresponds to the amount of independent regions, each one with a particular position and size, that are used by StarSim to model-fit the data. The StarSim fitting routine may group several of those elements on a single active region or spot group, so the fixed number of active surface elements does not determine the final number of active regions, but the maximum. While increasing the number of active elements may in principle improve the solution, constraining this parameter is a way of regularizing the solution and avoid fitting the noise present in the data. A certain amount of bright faculae around cool spot elements can also be included in the model, considering a hotter surface temperature and a limb brightening law as described by Meunier et al. (2010). The best solution for the stellar surface map is found by means of a simulated annealing approach (Herrero et al. 2016). The parameter space for some of the physical properties of the system can also be explored using StarSim and MCMC techniques (Rosich et al., in prep.). These were not used in the this work because of the computational cost of performing a large number of simulations of long time series, especially when the surface map is complex, as is the case for GJ1214. Instead, a more simple grid searh method was used. Finally, in the current version of StarSim, multiple time series data can be combined to provide a single solution for the stellar parameters and the surface map.

In this work, we fit for the surface map of GJ 1214 for the five seasons covered by the data described in Sect. 2 using StarSim and the parameters shown in Table 2. In StarSim, the limb darkening for each surface element is computed by interpolating intensities from Kurucz ATLAS 9 models. As these are truncated at $3500 \mathrm{~K}$, the intensities for GJ 1214 need to be extrapolated. However, the quadratic coefficients computed with StarSim for the three analyzed filters are in good agreement with the coefficients computed by Claret \& Bloemen (2011) using Phoenix models.

The stellar axis inclination is an input parameter for StarSim's simulations. As GJ 1214 is a very slow rotator, there are no well resolved $v \sin i$ measurements or observations of the Rossiter-McLaughlin effect. In this work we assume that the planet - star system is aligned, as is the case in other super-Earths (Barnes et al. 2015). Given that we fix the stellar axis inclination of GJ 1214 to $88.80_{-0.20}^{+0.25}$ (Berta et al. 2011), that is, nearly equator on, the solutions obtained with StarSim for the stellar surface map are degenerated in latitude, and only the recovered longitudes of the spot distribution and the projected filling factor of spots can be considered reliable.

In a first step of the modeling process, we performed a grid search by running 10000 solutions considering a simple surface model consisting of four active surface elements. This is done in order to find the best fit for the photometric zero points $\left(z_{\mathrm{B}}, z_{\mathrm{V}}\right.$ and $\left.z_{\mathrm{I}}\right)$ and the temperature contrast of the spots $\left(\Delta T_{\text {spots }}\right)$. The choice of four active elements in the first step of the StarSim modeling was made after trying solutions with two to ten elements, and finding that four was the minimum which successfully reproduced the light curve by visual inspection. While this is a first suggestion of the number of long-lived active regions in GJ 1214, the detailed evolution of the stellar surface map will be discussed after the second step of the modeling procedure. The photometric zero points are defined as $z_{i}=1 /\left\langle f_{i}\right\rangle$, where $f_{i}$ is the relative flux for filter $i$. A relative flux $f_{i}=1$ for $i=B, V, I$ is assumed for a completely quiet photosphere. A uniform grid of $100 \times 100$ different values for $\Delta T_{\text {spots }}$ and $z_{\mathrm{V}}$ is defined within the ranges $[0 \mathrm{~K}, 600 \mathrm{~K}]$ and [1.0, $1.25]$, respectively. Then, the $\chi^{2}$ for the best surface map solution is computed in each case considering all the multicolor information. The rest of the photometric zero points $\left(z_{\mathrm{B}}\right.$ and $\left.z_{\mathrm{I}}\right)$ are dependent on the two free parameters, $\Delta T_{\text {spots }}$ and $z_{\mathrm{V}}$. Therefore, these are used to compute $z_{\mathrm{B}}$ and $z_{\mathrm{I}}$ for each solution by simulating light curves with StarSim in $B$ and $I$ filters, considering the whole time span of the observed datasets. A weight scaled with $\sim \exp \left[-\left(\chi^{2} / \chi_{\text {scale }}^{2}\right)\right]$, with $\chi_{\text {scale }}^{2}=100$, is applied to each solution in order to produce the distributions displayed in Fig. 3. The purpose of such scaling is to define a distribution which presents a peak around the solutions with the lowest $\chi^{2}$. The median and the $68 \%$ confidence limits of the distributions (see Fig. 3) are adopted as the best results for $\Delta T_{\text {spots }}$ and $z_{\mathrm{V}}$. The Phoenix model grids used in StarSim are truncated at $2600 \mathrm{~K}$, thus preventing us to explore solutions for $\Delta T_{\text {spots }}>600 \mathrm{~K}$ in the case of GJ 1214. However, the result for the temperature contrast of the spots is in good agreement with the range of values observed for other M dwarfs (Berdyugina 2005). Instead of the more commonly used MCMC techniques, this type of analysis was chosen because of the computational cost of performing thousands of simulations with StarSim (see Herrero et al. 2016), given the length of the photometric time series and the complexity of the surface of GJ 1214. The results are shown in Table 2.

When multicolor time-series photometry is available, the variability amplitude differences allow for a measurement of the average temperature contrast of the active regions. The results from the first step of the StarSim modeling already show that the photometry can be modeled considering only cool spots with a temperature contrast around $\sim 400 \mathrm{~K}$. Therefore, while a certain number of faculae might be present in the active regions, our results show that the photometric variability on GJ1214 is dominated by the effects of cool spots. This is also supported by the fact that, while transit photometry of GJ 1214b has shown multiple spot-crossing events (Berta et al. 2011; Carter et al. 2011; Nascimbeni et al. 2015; Narita et al. 2013; Kreidberg et al. 2014), no signs of faculae features have been reported yet. Also, Youngblood et al. (2017) detected emission from GJ1214 at certain metal lines and also in $\mathrm{Ca} \mathrm{K}$, but among the $15 \mathrm{M}$ dwarfs of the MUSCLES survey (France et al. 2016; Youngblood et al. 2017), GJ1214 is at the lower end of UV emission line flux and it shows one order of magnitude less emission line flux in $\mathrm{Ca} \mathrm{K}$ than the 
Table 2. System parameters of GJ 1214.

\begin{tabular}{lcccc}
\hline \hline Parameter & Symbol & Value & Unit & Reference \\
\hline Effective temperature & $T_{\text {eff }}$ & $3252 \pm 20$ & $\mathrm{~K}$ & Anglada-Escudé et al. (2013) \\
Temperature contrast of spots & $\Delta T_{\text {spots }}$ & $372_{-182}^{+138}$ & $\mathrm{~K}$ & This work, fitted \\
Stellar surface gravity & $\log g$ & $5.01 \pm 0.07$ & - & Anglada-Escudé et al. (2013) \\
Facula to spot area ratio & $Q$ & 0.0 & - & This work, fixed \\
Rotation period & $P_{\text {rot }}$ & $125 \pm 5$ & days & This work, fitted \\
Planet orbit inclination & $i$ & $88.80_{-0.20}^{+0.25}$ & $\circ$ & Berta et al. (2011) \\
Planet orbital period & $P_{\text {planet }}$ & $1.58040482 \pm 0.00000024$ & days & Kreidberg et al. (2014) \\
Planet transit epoch & $t_{0}$ & $54966.52488 \pm 0.00004$ & days & Kreidberg et al. (2014) \\
Planet impact parameter & $b$ & $0.19_{-0.11}^{+0.08}$ & - & Berta et al. (2012a) \\
Planet to star radius ratio & $k$ & $0.1160 \pm 0.0005$ & - & Berta et al. (2012a) \\
Johnson $B$ photometric zero point & $z_{\mathrm{B}}$ & $1.06_{-0.04}^{+0.05}$ & - & This work, fitted \\
Johnson $V$ photometric zero point & $z_{\mathrm{V}}$ & $1.06_{-0.04}^{+0.05}$ & - & This work, fitted \\
Johnson $I$ photometric zero point & $z_{\mathrm{I}}$ & $1.04_{-0.04}^{+0.05}$ & - & This work, fitted \\
\hline
\end{tabular}
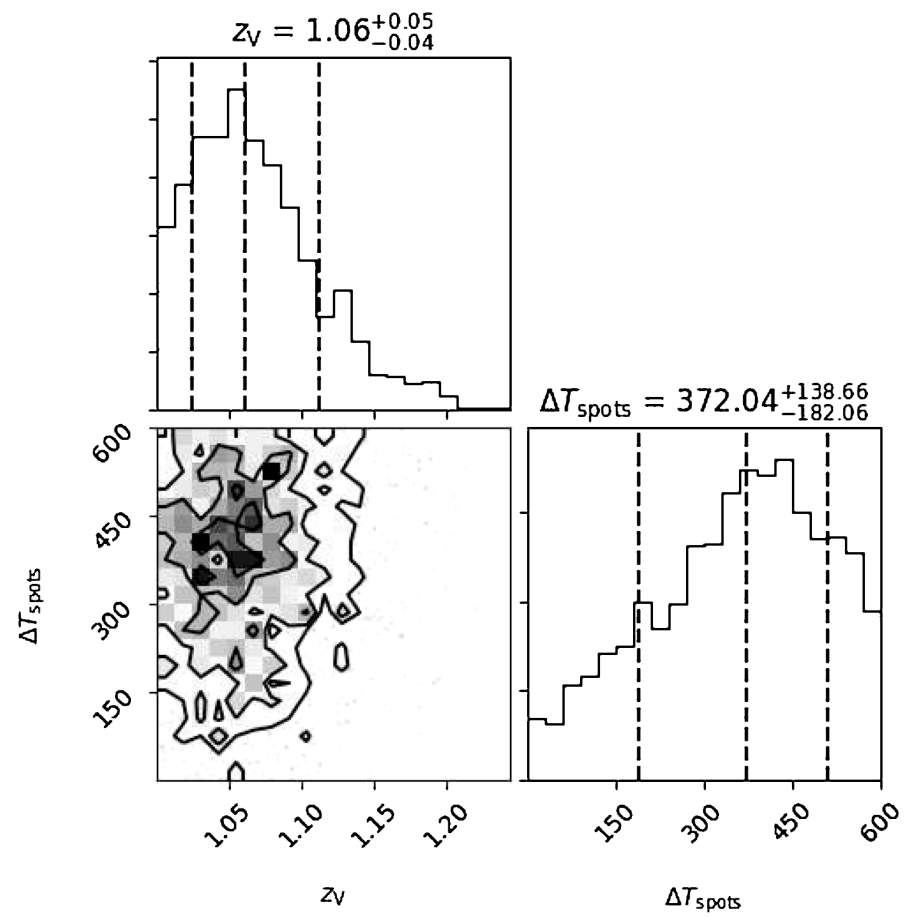

Fig. 3. Probability distribution of the photometric zero point in the Johnson $V$ band $\left(z_{\mathrm{V}}\right)$ and the temperature contrast of the spots $\left(\Delta T_{\text {spots }}\right)$. The vertical dashed lines indicate the median and the $68 \%$ confidence limits of the distributions.

rest of the targets. In order to avoid additional degeneracies in the models, in this work we assumed that the presence of faculae or bright regions is negligible, so the facula-to-spot area ratio is fixed to $Q=0$. In a recent paper Rackham et al. (2017) suggest that GJ 1214b transmission spectroscopy observations are modified by unocculted bright regions in the stellar photosphere. We discuss this further in Sect. 4.4.

In a second step of the fitting procedure with StarSim, we fixed the parameters from Table 2, including the starspot temperature contrast and the zeropoints found in the first step. So we only fitted for the number, the lifetimes, and the surface distribution of the active elements. Figure 4 displays the relative fluxes for the STELLA datasets in $B, V$ and $I$ filters, together with the best fit found with StarSim. The standard deviations of the residuals, in relative flux units, are $\sigma_{\mathrm{B}}=3.71 \times 10^{-3}$, $\sigma_{\mathrm{V}}=2.50 \times 10^{-3}$ and $\sigma_{\mathrm{I}}=3.36 \times 10^{-3}$ in $B, V$ and $I$ bands, respectively. These are near 1.7 times the standard error of the mean points for the three light curves. Figure 4 also displays the evolution of the projected filling factor of spots given by our best solution.

In order to estimate the properties of active regions during the time span covered by our data, the parameters from Table 2 were adopted and 256 solutions for the surface map were run with StarSim. The average map, projected to the longitude axis, is shown in Fig. 5, displaying the spot coverage probability vs. longitude and time. The light curves and also the projected filling factor of spots produced by these solutions will be nearly identical, while the surface maps can be slightly different given the existing degeneracies with the size and latitude of the spots. By averaging 256 solutions we obtained the surface map of the spot covering probability, preventing small short-lived spots from appearing on it. This is also a way of regularizing the solution and obtaining a smoother surface map that puts the focus on the main regions where spots are preferred by the model. The map displayed in Fig. 5 shows that there are at least four long lived active regions, including a dominant one which persists during all the time span covered with our photometry (more than four years). Also, all the active regions are stable in longitude in the reference frame of the stellar rotation period, indicating no signs of differential rotation.

\subsection{Spectral signature of active regions}

The atmospheres of exoplanets are often studied by using transmission spectroscopy techniques, in which low resolution spectroscopy is obtained during planetary transits in order to simultaneously measure the transit depth at multiple wavelengths. A slope toward the blue optical wavelengths seems to be present for most gas giants (Sing et al. 2016; Mallonn \& Wakeford 2017). A widely accepted interpretation is the presence of a cloud or haze layer (Wakeford \& Sing 2015; Pinhas \& Madhusudhan 2017). However, a unique physical interpretation of the spectral slope does not exist because spots on the host star can mimic the slope as well as third light from another stellar source along the line of sight (McCullough et al. 2014; Herrero et al. 2016).

In this work, we used the results from the modeling of the photometric time series described in Sect. 3.2 in order 


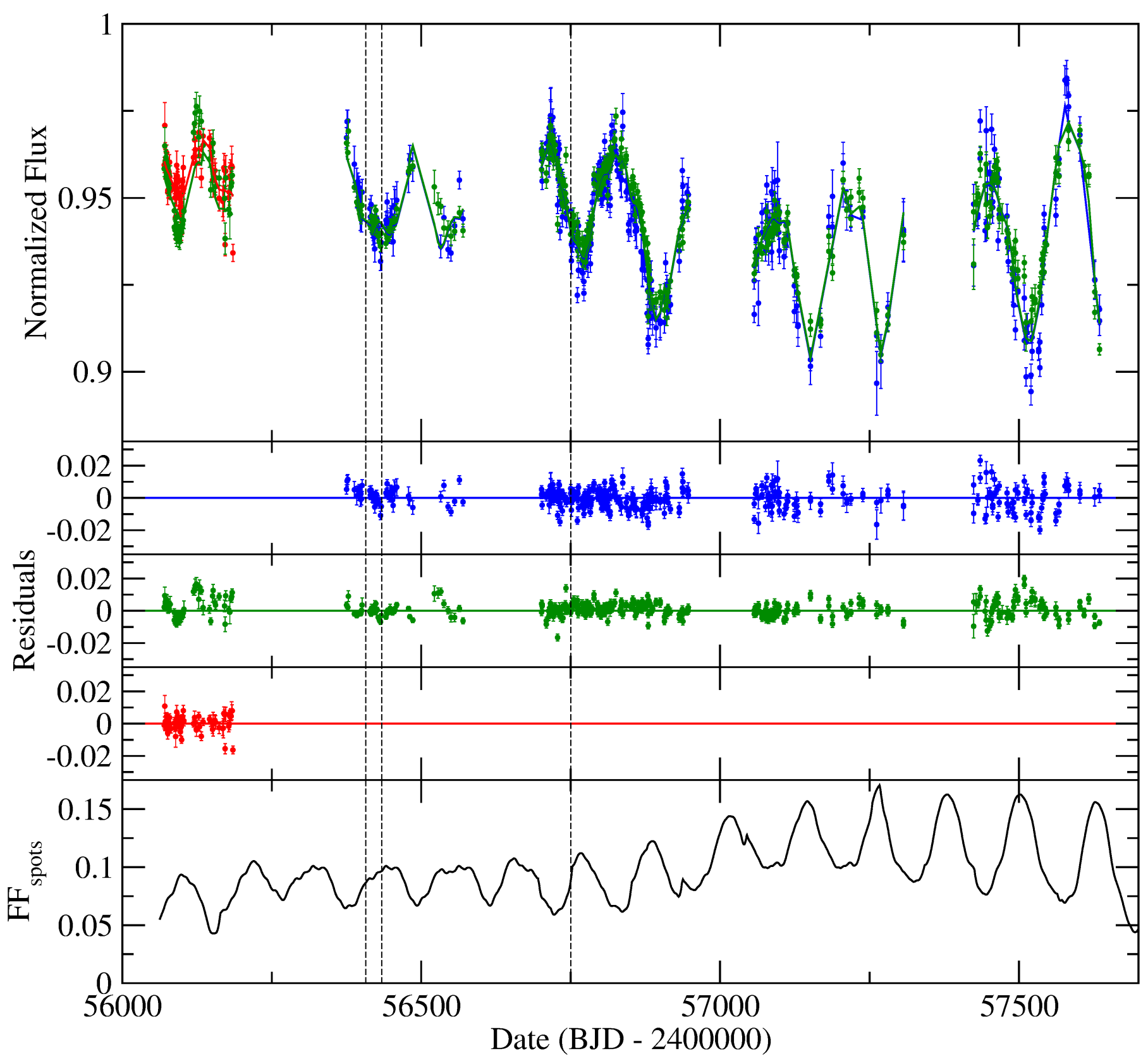

Fig. 4. Top panel: light curves of GJ 1214 from 2012 to 2016. Blue data points denote measurements in the Johnson $B$ filter, whereas green and red points indicate observations in the Johnson $V$ and Cousin $I$ filter, respectively. The photometric zero points are set according to the results presented in Table 2. The light curve models obtained with StarSim for the three bands are plotted with solid lines. Middle panels: residuals between the data and the model for the $B, V$, and $I$ filter (from top to bottom). Bottom panel: Evolution of the projected filling factor of spots for the best fitting model obtained with StarSim. The three vertical dashed lines in all panels indicate the mid times of the transits analyzed by Rackham et al. (2017).

to analyze a simulated series of transits of GJ $1214 \mathrm{~b}$ for the potential effect of the starspots on the planetary transmission spectrum. The procedure is analog to the one shown for HD189733 b by Herrero et al. (2016). While the degeneracies in our surface map solution, especially affecting the latitudes of the spots, prevent us from simulating specific transit events including all the particular effects of occulted and unocculted spots, we were able instead to simulate a large series of transits and analyze the statistics of the transmission spectra affected by a wide diversity of spot distributions. In the current work, the best solution for the stellar surface map, together with the stellar parameters listed in Table 2, were considered in order to generate series of simulated transits including the effects of cool spots on the stellar surface. Each series contains the 635 transits occurring during the time intervals covered by the photometry in Fig. 4. An additional transit was simulated considering the star with a completely unspotted surface with the purpose of computing precise limb darkening coefficients for each wavelength bin. A series of light curves was simulated for each transit event, covering the wavelength range from $400 \mathrm{~nm}$ to $2000 \mathrm{~nm}$ in bins of $50 \mathrm{~nm}$, so a transmission spectrum with 32 points is produced for each transit. The sections of the transits affected by spot crossing events were removed from the simulated light curves. This is done automatically by StarSim as an option when generating transit light curves, by using the information about the relative position 


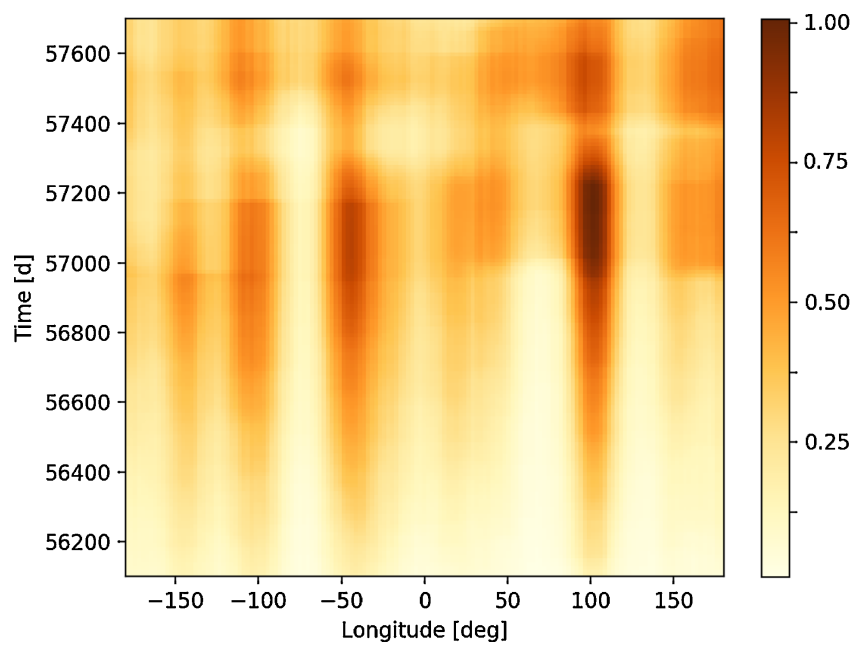

Fig. 5. Spot covering probability, displayed with different colour intensities from white (quiet photosphere) to dark brown (cool spots), versus time and longitude, for the average of 256 StarSim surface map solutions with the parameters fixed from Table 2 .

between the planet and the active regions at any time of the simulation.

The simulated series of transits were analyzed using JKTEBOP (Southworth et al. 2004; Southworth 2008). A square root limb darkening law was adopted, as this is known to be the optimal approximation for late type stars in the NIR (van Hamme 1993). Limb darkening coefficients, together with the sum of the radii and the planet to star radius ratio $\left(k=R_{\mathrm{p}} / R_{\mathrm{s}}\right)$, were kept as free parameters when fitting the unspotted transit of each passband. For all the simulated passbands, the computed limb darkening coefficients are in good agreement with those obtained by Claret (2000) for commonly used optical and NIR filters. Then, we fixed these coefficients for fitting the rest of the transit series, thus deriving the variations of $k$ produced by the presence of unocculted spots. For each transit $i$, the transit depth variation $\Delta k_{\mathrm{i}}=k_{\mathrm{i}}-k_{\text {unspotted }}$ was obtained, with $k_{\text {unspotted }}$ being the planet to star radius ratio of the unspotted transit, adopted as reference. Finally, we computed the mean and standard deviation of $\Delta k$ for the 635 transits in each wavelength bin. These are displayed in Fig. 6, thus showing the chromatic signature of spots on transit depth measurements. Such signature is strongly dependent on the amount of spots and their temperature contrast $\left(\Delta T_{\text {spots }}\right)$. Therefore, only by simultaneously analyzing chromatic information (i.e., multiple wavelength photometry) we could recover accurate properties of spots and their chromatic effects on transmission spectroscopy measurements. Mean $\Delta k$ values and $68 \%$ confidence levels are also plotted in Fig. 6 for all the analyzed spectral range. The spectral signature of spots on $\Delta k$ was also computed for the transits occurring in seasons 2013 and 2014 without removing the effects off spot crossing events from the simulated light curves. This is displayed in the bottom panel of Fig. 6 . The bumps introduced by occulted spots in the transit light curves make the distribution of $\Delta k$ to deviate from a Gaussian, thus causing the mean $\Delta k$ values to be close to the upper $68 \%$ confidence level.

According to our results from simulating transit depth variations, the spots present on the surface of GJ 1214 can bias the planet to star radius ratio by up to $7 \times 10^{-3}$ in the blue optical wavelengths and up to $3 \times 10^{-3}$ in the NIR. In all our simulations where spot occultation effects were removed there is a positive $\Delta k$ due to a permanent spot coverage in GJ 1214 (see Fig. 5).

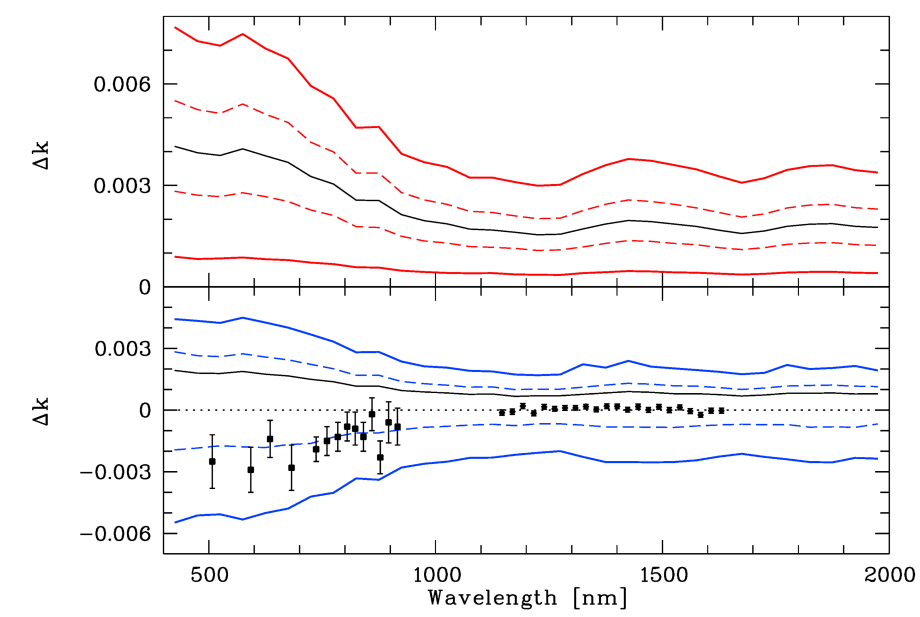

Fig. 6. Modifications of the planet to star radius ratio by spots. The upper plot shows the variations $\left(\Delta k=k-k_{\text {unspotted }}\right)$ with wavelength, which we derived from analyzing the 635 simulated transits in 32 wavelength bins. The black line indicates the mean values of $\Delta k$ for each wavelength bin with the dashed red line indicating the $68 \%$ confidence interval. The solid red lines indicate the region between the minimum and maximum $\Delta k$. In the bottom plot, we show the same for the 278 transits occurring during seasons 2013 and 2014, without removing spot crossing features. The blue lines indicate the region between the minimum and maximum $\Delta k$. The overplotted optical measurements are from Rackham et al. (2017), the NIR data are from Kreidberg et al. (2014).

In transmission spectroscopy, we are interested in the relative change of $\Delta k$ over wavelength instead of its absolute value. This relative change would manifest as a spectral slope. At the maximum of the spot filling factor in the year 2015 it amounts to an amplitude of $(4.3 \pm 1.6) \times 10^{-3}$ between 500 and $1200 \mathrm{~nm}$, while for an average filling factor between 2012 and 2016 it amounts to $(2.5 \pm 0.9) \times 10^{-3}$. This is a factor of 2.5 above the typical uncertainty of a measurement of $k$ at blue wavelengths (de Mooij et al. 2013; Nascimbeni et al. 2015). Therefore, the influence of unocculted spots in the optical is not negligible during a phase of large photometric variations. We note that the majority of transit observations for transmission spectroscopy of GJ 1214b have been taken from 2009 to 2012, where the star might have had a significantly lower spot coverage due to a magnetic activity cycle (see Sect. 4.2). The most precise transmission spectrum of GJ 1214b was derived from 12 transit observations with the HST by Kreidberg et al. (2014), observed between September 2012 and August 2013. Our simulations show that their covered wavelength range from 1100 to $1700 \mathrm{~nm}$ is generally less affected by spots and shows only smooth variations.

\section{Discussion}

\subsection{Variability in the context of MEarth M dwarfs}

The exoplanet of GJ 1214 was discovered by the ground-based transit survey MEarth, which monitors several thousands of midto-late $\mathrm{M}$ dwarfs to search for transiting planets (Berta et al. 2012b). Newton et al. (2016b) analyzed the variability amplitude and periodicity of $387 \mathrm{M}$ dwarfs of the MEarth northern target list. To place GJ 1214 in this context, we compared the rotation period and variability amplitude derived here for GJ 1214 with those of the Newton sample, which did not include our target. In Fig. 7 we reproduce the mass-period diagram, showing that lower-mass stars spin down to longer rotation periods than higher-mass stars, a result already reported in Irwin et al. (2011) 
and McQuillan et al. (2014). The rotation period of GJ 1214 of 125 days is among the longest periods found, but is well within the upper envelope of detected rotation periods. The longest periods found by Newton et al. (2016b) are about 140 days. GJ 1214 covers the same parameter space in this diagram as the other two planet host stars found by the MEarth survey, GJ 1132 (Berta et al. 2015) and LHS 1140 (Dittmann et al. 2017).

In the next step, we reproduced the period-amplitude diagram of stars less massive than 0.25 solar masses of Newton et al. (2016b). For GJ 1214 we transformed the peak-to-peak variability amplitude of $5.3 \%$ in Johnson $V$ to a semi-amplitude in the MEarth filter of about $1.6 \%$. This transformation was done by simulating the light curve from 2012 to 2016 in the MEarth passband with StarSim, using the parameters found in our analysis (see Table 2). The overall system response of an MEarth telescope is taken from (Nutzman \& Charbonneau 2008), the MEArth filter resembles approximately a combination of Sloan $i$ and $z$. In the period-amplitude diagram Fig. 8 we see GJ 1214 to be among the most variable long-period M dwarfs, strengthening the result of Newton et al. (2016b) that there is no anti-correlation of period and variability amplitude for low-mass M dwarfs. GJ 1132 and LHS 1140 show lower photometric variability by a factor of three. We note that Kado-Fong et al. (2016) lists several low-mass $\mathrm{M}$ dwarfs with rotation periods longer than 100 days and even higher variability amplitudes than GJ 1214 in the slightly redder Pan-STARRS1 $z$-band.

In HARPS spectra used for radial velocity measurements in the discovery paper (Charbonneau et al. 2009), no activity induced chromospheric emission in either the $\mathrm{H} \alpha$ or the NaI doublet was detected. Very weak emission in Ca II K was detected by Youngblood et al. (2017). Newton et al. (2017) derived a relation between stellar rotation period and chromospheric activity for M dwarfs of the MEarth sample, by which chromospherically inactive stars of 0.15 solar masses should have rotation periods of longer than 80 days. This derivation contradicts previously estimated rotation periods of GJ 1214 of 40 or 53 days (Narita et al. 2013; Berta et al. 2011). Our new derivation of a stellar rotation period of $125 \pm 5$ days agrees well with this activity-rotation relationship. In the same work, Newton et al. (2017) described a correlation between chromospheric activity and amplitude of photometric variability. The spectroscopic HARPS observations from Charbonneau et al. (2009) were taken in the year 2009. when GJ 1214 was at a state of lower photometric variability (see Sect. 4.2). Spectroscopic observations of the year 2014 or later would be needed to verify if this activity-variability trend also applies to GJ 1214. According to this, the increase in photometric amplitude measured in this work might be accompanied by an increase in chromospheric activity.

\subsection{An activity cycle for GJ 1214?}

The magnetic activity of the Sun undergoes a cyclic behavior which observationally manifests in the $\sim 11 \mathrm{yr}$ sunspot cycle. Observations of $\mathrm{F}$ to early $\mathrm{M}$ dwarfs indicate that a significant fraction of these solar-type stars displays similar cycles (Oláh et al. 2009; Strassmeier 2009; Suárez Mascareño et al. 2016). Stars later than spectral type $\sim$ M3.5 are expected to have fully convective interiors and therefore a different dynamo process responsible for magnetic activity than the earlier type solar-like stars (Charbonneau 2014). The question of whether or not fully convective stars show a cyclic behavior of magnetic activity is still open, but recent observational work points toward this possibility. The M6 dwarf Proxima Centauri shows an approximately seven year photometric variability indicative of

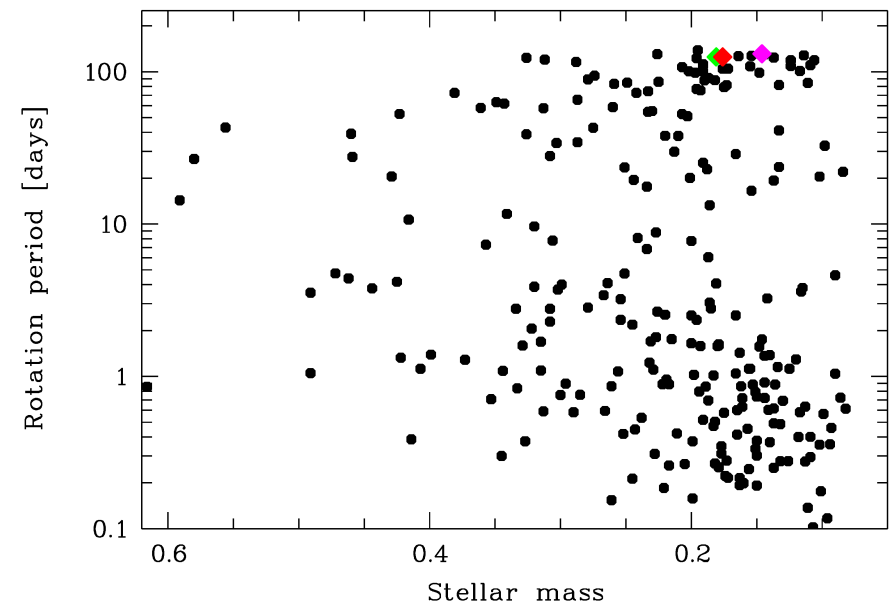

Fig. 7. Mass-period diagram of MEarth targets. Black data points are taken from Newton et al. (2016b), GJ 1214 is shown in red, GJ1132 and LHS1140 in green and purple, respectively. The rotation period of GJ 1214 of $125 \pm 5$ days matches the upper envelope of the distribution of increasing rotation periods with increasing stellar mass.

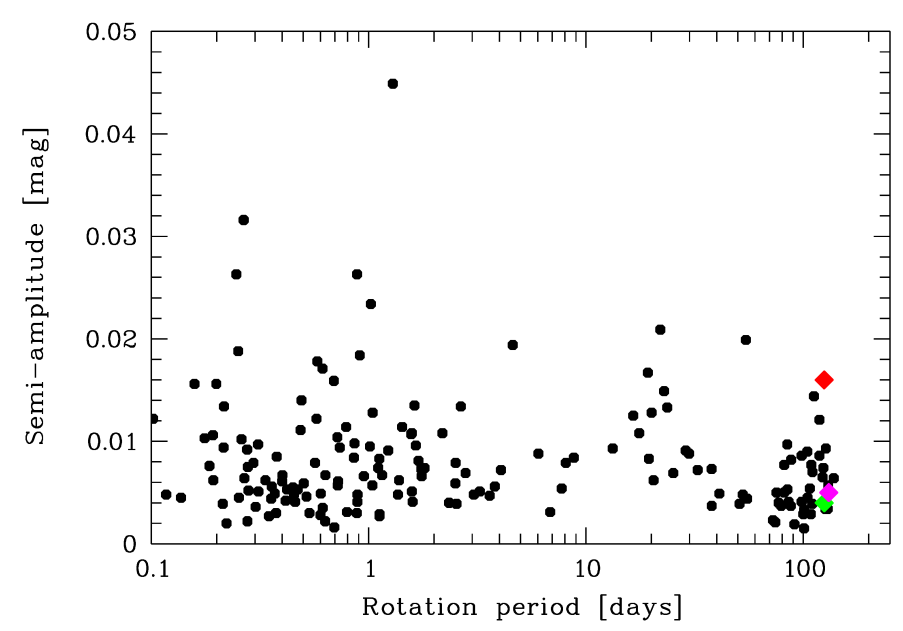

Fig. 8. Rotation-amplitude diagram of MEarth targets of masses below $0.25 M_{\odot}$. Black data points are taken from Newton et al. (2016b), GJ 1214 is shown in red, GJ 1132 and LHS 1140 in green and purple, respectively. GJ 1214 is one of the most variable stars among the group of very slow rotators. The result of Newton et al. (2016b) of no correlation between rotation and photometric variability amplitude is strengthened.

an activity cycle (Wargelin et al. 2017). In another work, Hosey et al. (2015) present four fully convective M dwarfs with cyclic photometric behavior from four to eight years period. Suárez Mascareño et al. (2016) found cycles from four to ten years for nine M4 to M6 dwarf stars.

The lower panel of Fig. 4 shows an increase of the spot filling factor for GJ 1214 from 2012 to 2015 . Additionally, we see a steady increase in amplitude of variability in our STELLA data, starting from a full-amplitude in $V$ of $\sim 2.5 \%$ in 2012 up to $5.3 \%$ in 2016. Transformed to the MEarth filter, these two values correspond to $1.4 \%$ and $3.2 \%$ of full amplitude. Berta et al. (2011) presented MEarth photometry from 2008 to 2010 with photometric full amplitudes of below $1 \%$. For the years prior to our monitoring campaign, it is unclear whether the increase in variability amplitude of GJ 1214 is driven by an overall increase in activity, potentially caused by a cyclic behavior of the stellar activity. Another reason for a larger variability amplitude might 
be a change in spot distribution toward less homogeneity compared to previous years. The shorter periods derived by previous studies might indicate the presence of more than one dominating spot group in 2010 and therefore a more homogeneous spot distribution than in 2013-2016. The StarSim light curve modeling of the STELLA data in this work suggests no significant changes in the spot distribution from 2012 to 2016 . Instead, several active longitudes remained stable over most of the observed time, but the spots grew in size. Therefore, the increase in variability in the STELLA data can be interpreted as a cycle of more than four years length. Solely comparing the photometric variability amplitude from 2008 to 2016, it appears that the cycle might be longer than eight years.

\subsection{On the age of GJ 1214}

The M dwarf GJ 1214 shows no sign of youth, for example, it shows no or very weak chromospheric line emission (Charbonneau et al. 2009; Youngblood et al. 2017). Low-mass stars spin down as they age, thus in principle we can use the stellar rotation period derived in this work as an age indicator. However, the quantitative framework exploiting this effect called gyrochronology (Barnes 2003, 2010) has not yet been empirically anchored for fully convective $\mathrm{M}$ dwarfs. Therefore we only use qualitative arguments here. Newton et al. (2016b) use age-space velocity relationships to estimate a mean age for the population of slowly rotating solar neighborhood mid $\mathrm{M}$ dwarfs of $5_{-2}^{+4}$ Gyr. The Alpha Cen system is believed to be somewhat older than the Sun, about 5.6 Gyr old (DeWarf et al. 2010), harboring the mid $\mathrm{M}$ dwarf Proxima Centauri with a rotation period of 83 days (Benedict et al. 1998). Barnard's star is suggested to be a very old, thick disk star, potentially exceeding $10 \mathrm{Gyr}$ (Choi et al. 2013) with a tentatively detected long stellar rotation period of about 130 days (Benedict et al. 1998). GJ 1214 is likely to be older than Proxima Centauri and as old or slightly younger as Barnard's star. Thus we conclude GJ 1214 to have an age of between 6 and $10 \mathrm{Gyr}$.

\subsection{Optical slope in the planetary transmission spectrum}

Rackham et al. (2017) observed three transit events in the years 2013 and 2014 and found an offset in the spectrum between optical and NIR wavelengths, in contrast to the optical results of Nascimbeni et al. (2015), de Mooij et al. (2013), and Narita et al. (2013). The observations for the latter three works were taken in the years 2011 and 2012. The tentative downward slope toward shorter wavelengths is consistently present in all three data sets of Rackham et al. (2017), and their favored interpretation are modifications of a flat planetary spectrum by unocculted bright regions in the stellar photosphere. In this interpretation, the stellar photosphere is either covered only with bright regions without dark spots, or the effect of the bright regions dominates. Here, we provide arguments for why we consider this scenario to be unlikely.

As presented in Sect. 3.2, the light curve inversion resulted in active regions that are around $400 \mathrm{~K}$ cooler than the quite photosphere. The long-term photometry of this work can be modeled solely by dark regions without any need for a more complex mixture of dark and bright regions. The temperature contrast between quiet photosphere and active surface elements is mainly derived by the multicolor information of the long-term variability, and the light curve inversion provides no indications for bright regions dominating this variability.
There is evidence of dark spots on the stellar photosphere of GJ 1214 by spot crossing events in transit observations from 2009 to 2013 (Berta et al. 2011; Carter et al. 2011; Nascimbeni et al. 2015; Narita et al. 2013; Kreidberg et al. 2014), and 2017 (Juvan et al. in prep.). The spot crossing events in two transits of Kreidberg et al. (2014) from August 2013 happened chronologically in between the transit observations of Rackham et al. (2017) in 2013 and 2014. The dominating spot groups are longliving (see Sect. 3.2), thus it is reasonable to assume that spots were always present in the photosphere of GJ 1214 between 2009 and 2017. However, none of the numerous published transit light curves showed evidence for a crossing of bright regions.

In a very recent study, Juvan et al. (in prep.) analyzes 25 transit light curves observed in the year 2017 together with simultaneous photometric monitoring. Spot crossing events have been found in the transit light curves only at times of a relative brightness dimming of the host star, indicative for more spots on the surface at times when the star is faint and therefore a spot dominated stellar variability.

The transit observations of Rackham et al. (2017) overlap with the photometric monitoring presented here. The epochs of the three transits are marked as vertical dashed lines in Fig. 4. At each transit, the star is near a minimum of relative flux. Hence, to be potentially responsible for the measured transmission spectrum, bright regions had to dominate over dark regions even at times of stellar flux minimum.

There are no indications for chromospheric activity of GJ 1214. If similar to the Sun, bright regions should be associated with chromospheric activity, but no - or very weak - line emission has been measured at $\mathrm{H} \alpha, \mathrm{Ca} \mathrm{K}$ and in the UV iron lines (Youngblood et al. 2017).

To summarize, there is no independent indication for the presence of bright regions in the photosphere of GJ 1214. Therefore we consider it as rather unlikely that bright regions could have been dominating over dark spots in modifying the transmission spectrum. In the following, we present an alternative explanation for the transmission spectrum measured by Rackham et al. (2017).

Assuming a flat optical planetary spectrum (strongly suggested by the bulk of published transmission spectroscopy results), a potential explanation for the measurement of a negative $\Delta k$ is the occultation of spots (Herrero et al. 2016). Spot crossing events during transits, if not properly modeled or removed, will produce negative results for $\Delta k$, especially in the blue optical wavelengths, where the contrast of the spots is higher (Pont et al. 2013; Oshagh et al. 2014; Herrero et al. 2016). Although our long-term photometry provides a spot filling factor for the times of the transits of Rackham et al. (2017), the surface distribution of spots are degenerated in latitude. Therefore, we cannot realistically simulate spot occultation events for these specific transits.

Instead, we simulated and analyzed all the transits that occurred during seasons 2013 and 2014, which also included the transit events observed by Rackham et al. (2017). We did this without removing the spot crossing features, in order to produce the statistics of $\Delta k$ variations when the projected filling factor of spots is $0.05-0.1$ and the spot crossing features are not properly removed from the transit photometry. The results from our analysis of the 278 transits simulated for seasons 2013 and 2014 are displayed in the bottom panel of Fig. 6, showing the spurious negative slope toward the blue wavelengths produced by spot crossing features. Overplotted are the transmission spectroscopy measurements of Rackham et al. (2017), which fall 
within the region of minimum and maximum $\Delta k$ as derived from the simulations.

No spot crossing signatures are photometrically resolved in the transit light curves of Rackham et al. (2017). Our transit simulations indicate that their photometric accuracy is sufficient to rule out the full occultation of a spot of the same size as the planet or larger. However, the complexity of the surface map during these seasons, showing a number of small active regions, could make small spot crossing features undetectable in the observed light curves. As shown in Fig. 5, the surface of GJ1214 exhibited a complex activity pattern during the years 2013 and 2014 (BJD from 56300 to 57000 ), consisting of big persisting spot groups and a large number of small spots spread over a wide range of longitudes. The analysis of the transit depth variations from our simulations show that the deviations in the measurements of $k$ due to spot crossing events can be of up to $5 \times 10^{-3}$, when three to four small spots are occulted. In the analyzed transit simulations, such small spots produce a relative flux increase in-transit of below $3 \times 10^{-3}$ in $B$ filter, while the transits observed by Rackham et al. (2017) show residuals of up to $5 \times 10^{-3}$ in relative flux in the blue wavelengths. Therefore, such transit observations could include small spot crossing features producing a $\Delta k$ of $-3 \times 10^{-3}$ as observed by Rackham et al. (2017). That could explain the observed chromatic trend in transit depth. However, we emphasize again that no spot crossing event was photometrically detected in the Rackham et al. (2017) transits. Therefore, the presented option of small crossed star spots can only be one viable possibility, rather than the final explanation of the measured transmission spectrum.

The case of the three transit observations of Rackham et al. (2017) illustrates the difficulty to measure the slope in a planetary transmission spectrum if the host star is very active. Our long-term photometry, in principle, allows for a correction of unocculted spots. However, we interpret the Rackham et al. (2017) measurements as being affected by unocculted and occulted spots at the same time. In this case, the solution is highly degenerate and no correction of the transmission spectrum for spots is possible anymore. The only solution would be a higher photometric precision per transit measurements to photometrically resolve the in-transit star spot crossing features. Unfortunately, typical ground-based transit observations in lowresolution spectroscopy do not reach the photon-noise limited precision (Mallonn \& Strassmeier 2016; Huitson et al. 2017). Many authors observe multiple transit events of an individual system to verify the robustness of results and increase the precision of the derived planetary transmission spectrum (Lendl et al. 2016; Gibson et al. 2017). However, without sufficient $\mathrm{S} / \mathrm{N}$ per individual data set, this approach does not necessarily increase the accuracy of the transmission spectrum because it might not allow us to differentiate effects of unocculted and occulted star spots by the photometric resolution of spot crossing features. Especially for M dwarfs the photometric detection tend to be burdensome. Their star spots often exhibit a low temperature contrast and produce only small spot bumps at red wavelengths. At blue wavelengths, where the spot bump is somewhat larger, their photometric resolution is difficult because of the low number of photons emitted by the host star.

In the case of photometrically resolved spot bumps, we suggest the simultaneous fitting of a transit model, the in-transit spot bump as well as correlated noise. Examples of capable software tools are spotrod (Béky et al. 2014), PRISM (TregloanReed et al. 2013), and SOAP-T (Oshagh et al. 2013a). Recently, Juvan et al. (2018) developed PyTranSpot, which allows for the simultaneous analysis of multicolor light curves of active stars.
The presence of faculae has a very strong impact on the radial velocity signature, both on amplitude and shape, as they are regions where convection is blocked. Herrero et al. (2016) was able to constrain the facula-to-spot area ratio for HD 189733 to be close to zero by the analysis of simultaneous photometric and radial velocity monitoring. We suggest the observation of similar data for GJ 1214 to constrain $Q$.

\section{Conclusion}

We presented a photometric monitoring campaign over five seasons of GJ 1214 as part of the host stars monitoring survey VAMOS. In the observed period, the mid M dwarf, which hosts a super-Earth planet, showed significant photometric variations due to active regions rotating in and out of view. The data reveal a clear signal in the calculated periodogram at $125 \pm 5$ days, which we interpret as the stellar rotation period. Our result does not confirm previous measurements of the rotation period. The period of GJ 1214 is not unusual for a fully convective M dwarf of 0.176 solar masses. When compared to other northern MEarth targets from Newton et al. (2016b), it matches with the upper envelope of the mass-period distribution. GJ 1214 is among the most variable objects of the slow rotators, confirming the result of Newton et al. (2016b) of no correlation between rotation period and photometric variability amplitude for star of lower mass than 0.25 solar masses.

We confirm the finding of Newton et al. (2017) that low-mass stars, such as GJ 1214, without chromospheric emission have periods longer than 80 days. Previous claims on a shorter rotation period of GJ 1214 contradicted this result. Newton et al. (2017) also described a positive correlation of photometric variability with chromospheric line emission. New spectroscopy of $\mathrm{H} \alpha$ would be needed to verify if the measured increase in photometric variability is accompanied by an increase in chromospheric emission when compared to the non-detection of activity by Charbonneau et al. (2009).

We modeled the five-year multicolor photometry with the light curve inversion tool StarSim (Herrero et al. 2016) and derived the color dependent photometric zero points, defining the relative flux level of an unspotted photosphere. We find that GJ 1214 was on average dimmed by star spots by $6 \%$ in Johnson $B$ and Johnson $V$, and by $4 \%$ in Cousins $I$. Furthermore we derived a star spot contrast temperature of $370 \mathrm{~K}$, in agreement with previous results of weaker spot contrasts for late type stars than for solar type stars (Berdyugina 2005). The variation is stable in phase over four years from 2013 to 2016, being caused by long-living active longitudes. The values of the longitudes remain stable over time within the measurement precision, thus we find no indications for differential rotation in the stellar photosphere. We measured an increase in amplitude of photometric variability over the time interval covered by our observations. Also the light curve inversion with StarSim yields an increasing spot filling factor. This can be interpreted as an activity cycle similar to the 11-year cycle of the Sun. The existence of such cyclic behavior in fully convective stars is still an open question. The data presented in this work point toward a cycle length of more than four years. If we compare the photometry of this work with MEarth photometry of the years 20082010, the variability roughly monotonically increased from 2008 to 2016, being indicative of a cycle length of more than eight years.

From the StarSim modeling we were able to derive a surface map of the stellar photosphere for all 635 transit epochs between the beginning and end of our five seasons of 
monitoring. We used these maps to produce the associated transit light curve from 400 to $2000 \mathrm{~nm}$ with StarSim. These light curves, all affected by a certain amount of spots, were fitted with a transit light curve model. This exercise resulted in values for the mean, minimum and maximum star spot modification of the transit depth by unocculted star spots over wavelength. In principle, this exercise provides the wavelength-dependent correction of transit depth for unocculted spots for any transit epoch during the observing campaign.

The effect of unocculted spots on the transit depth is always positive, meaning that the depth of transit is enhanced. Rackham et al. (2017) observed three transit events in 2013 and 2014 and measured a slightly shallower transit depth at blue optical wavelengths than previous studies. Our photometry shows their transit epochs to be close to photometric minima, making it rather unlikely that the shallow transit depth is caused by unocculted bright regions. Another viable explanation for a shallower transit depth are occulted dark spots hidden in the photometric noise of the transit light curve. However, despite having simultaneous long-term photometry on hand, we cannot correct for occulted spots when their signature is not photometrically resolved. This makes it impossible to break the degeneracy in the optical slope of a derived transmission spectrum between spot effects and planetary spectral signature for active host stars.

Acknowledgements. E.H., A.R. and I.R. acknowledge support by the Spanish Ministry of Economy and Competitiveness (MINECO) and the Fondo Europeo de Desarrollo Regional (FEDER) through grant ESP2016-80435-C2-1-R, as well as the support of the Generalitat de Catalunya/CERCA program. I.G.J. acknowledges the Austrian Forschungsförderungsgesellschaft FFG project "RASEN" P847963. This research has made use of the SIMBAD data base and VizieR catalog access tool, operated at CDS, Strasbourg, France, and of the NASA Astrophysics Data System (ADS).

\section{References}

Allard, F., Homeier, D., \& Freytag, B. 2011, in 16th Cambridge Workshop on Cool Stars, Stellar Systems, and the Sun, eds. C. Johns-Krull, M. K. Browning, \& A. A. West, ASP Conf. Ser., 448, 91

Anglada-Escudé, G., Rojas-Ayala, B., Boss, A. P., Weinberger, A. J., \& Lloyd, J. P. 2013, A\&A, 551, A48

Barnes, J. W., Ahlers, J. P., Seubert, S. A., \& Relles, H. M. 2015, ApJ, 808, L38

Barnes, S. A. 2003, ApJ, 586, 464

Barnes, S. A. 2010, ApJ, 722, 222

Barstow, J. K., Aigrain, S., Irwin, P. G. J., Kendrew, S., \& Fletcher, L. N. 2015, MNRAS, 448, 2546

Bean, J. L., Miller-Ricci Kempton, E., \& Homeier, D. 2010, Nature, 468, 669

Bean, J. L., Désert, J.-M., Kabath, P., et al. 2011, ApJ, 743, 92

Béky, B., Kipping, D. M., \& Holman, M. J. 2014, MNRAS, 442, 3686

Benedict, G. F., McArthur, B., Nelan, E., et al. 1998, AJ, 116, 429

Berdyugina, S. V. 2005, Liv. Rev. Sol. Phys., 2, 8

Berta, Z. K., Charbonneau, D., Bean, J., et al. 2011, ApJ, 736, 12

Berta, Z. K., Charbonneau, D., Désert, J.-M., et al. 2012a, ApJ, 747, 35

Berta, Z. K., Irwin, J., Charbonneau, D., Burke, C. J., \& Falco, E. E. 2012b, AJ, 144,145

Berta, Z. K., Irwin, J., Charbonneau, D., et al. 2015, Nature, 527, 204

Bertin, E. \& Arnouts, S. 1996, A\&AS, 117, 393

Carter, J. A., Winn, J. N., Holman, M. J., et al. 2011, ApJ, 730, 82

Castelli, F. \& Kurucz, R. L. 2004, Proc. IAU Symp., 210, poster A20
Charbonneau, D., Berta, Z. K., Irwin, J., et al. 2009, Nature, 462, 891 Charbonneau, P. 2014, ARA\&A, 52, 251

Choi, J., McCarthy, C., Marcy, G. W., et al. 2013, ApJ, 764, 131 Claret, A. 2000, A\&A, 363, 1081

Claret, A. \& Bloemen, S. 2011, A\&A, 529, A75

Croll, B., Albert, L., Jayawardhana, R., et al. 2011, ApJ, 736, 78 de Mooij, E. J. W., Brogi, M., de Kok, R. J., et al. 2013, ApJ, 771, 109 DeWarf, L. E., Datin, K. M., \& Guinan, E. F. 2010, ApJ, 722, 343 Dittmann, J. A., Irwin, J. M., Charbonneau, D., et al. 2017, Nature, 544, 333 Fortney, J. J., Shabram, M., Showman, A. P., et al. 2010, ApJ, 709, 1396 France, K., Parke Loyd, R. O., Youngblood, A., et al. 2016, ApJ, 820, 89

Gibson, N. P., Nikolov, N., Sing, D. K., et al. 2017, MNRAS, 467, 4591 Gillon, M., Demory, B.-O., Madhusudhan, N., et al. 2014, A\&A, 563, A21 Granzer, T., Weber, M., \& Strassmeier, K. G. 2010, Advances in Astronomy, 2010,980182

Herrero, E., Ribas, I, Jordi, C., et al. 2016, A\&A, 586, A131

Hosey, A. D., Henry, T. J., Jao, W.-C., et al. 2015, AJ, 150, 6

Huitson, C. M., Désert, J.-M., Bean, J. L., et al. 2017, AJ, 154, 95

Irwin, J., Berta, Z. K., Burke, C. J., et al. 2011, ApJ, 727, 56

Juvan, I. G., Lendl, M., Cubillos, P. E., et al. 2018, A\&A, 610, A15

Kado-Fong, E., Williams, P. K. G., Mann, A. W., et al. 2016, ApJ, 833, 281

Kirk, J., Wheatley, P. J., Louden, T., et al. 2017, MNRAS, 468, 3907

Kreidberg, L., Bean, J. L., Désert, J.-M., et al. 2014, Nature, 505, 69

Lendl, M., Delrez, L., Gillon, M., et al. 2016, A\&A, 587, A67

Mallonn, M., \& Strassmeier, K. G. 2016, A\&A, 590, A100

Mallonn, M., \& Wakeford, H. R. 2017, Astron. Nachr., 338, 773

Mallonn, M., Nascimbeni, V., Weingrill, J., et al. 2015a, A\&A, 583, A138

Mallonn, M., von Essen, C., Weingrill, J., et al. 2015b, A\&A, 580, A60

McCullough, P. R., Crouzet, N., Deming, D., \& Madhusudhan, N. 2014, ApJ, 791, 55

McQuillan, A., Mazeh, T., \& Aigrain, S. 2014, ApJS, 211, 24

Meunier, N., Desort, M., \& Lagrange, A.-M. 2010, A\&A, 512, A39

Miller-Ricci, E. \& Fortney, J. J. 2010, ApJ, 716, L74

Narita, N., Fukui, A., Ikoma, M., et al. 2013, ApJ, 773, 144

Nascimbeni, V., Mallonn, M., Scandariato, G., et al. 2015, A\&A, 579, A113

Newton, E. R., Irwin, J., Charbonneau, D., Berta-Thompson, Z. K., \& Dittmann, J. A. 2016a, ApJ, 821, L19

Newton, E. R., Irwin, J., Charbonneau, D., et al. 2016b, ApJ, 821, 93

Newton, E. R., Irwin, J., Charbonneau, D., et al. 2017, ApJ, 834, 85

Nutzman, P., \& Charbonneau, D. 2008, PASP, 120, 317

Oláh, K., Kolláth, Z., Granzer, T., et al. 2009, A\&A, 501, 703

Oshagh, M., Boisse, I., Boué, G., et al. 2013a, A\&A, 549, A35

Oshagh, M., Santos, N. C., Boisse, I., et al. 2013b, A\&A, 556, A19

Oshagh, M., Santos, N. C., Ehrenreich, D., et al. 2014, A\&A, 568, A99

Pinhas, A., \& Madhusudhan, N. 2017, MNRAS, 471, 4355

Pont, F., Sing, D. K., Gibson, N. P., et al. 2013, MNRAS, 432, 2917

Rackham, B., Espinoza, N., Apai, D., et al. 2017, ApJ, 834, 151

Sedaghati, E., Boffin, H. M. J., MacDonald, R. J., et al. 2017, Nature, 549, 238

Sing, D. K., Fortney, J. J., Nikolov, N., et al. 2016, Nature, 529, 59

Southworth, J. 2008, MNRAS, 386, 1644

Southworth, J., Maxted, P. F. L., \& Smalley, B. 2004, MNRAS, 351, 1277

Strassmeier, K. G. 2009, A\&ARv, 17, 251

Strassmeier, K. G., Granzer, T., Weber, M., et al. 2004, Astron. Nachr., 325, 527

Suárez Mascareño, A., Rebolo, R., \& González Hernández J. I. 2016, A\&A, 595, A12

Teske, J. K., Turner, J. D., Mueller, M., \& Griffith, C. A. 2013, MNRAS, 431, 1669

Tregloan-Reed, J., Southworth, J., \& Tappert, C. 2013, MNRAS, 428, 3671

van Hamme W. 1993, AJ, 106, 2096

von Essen, C., Cellone, S., Mallonn, M., et al. 2017, A\&A, 603, A20

Wakeford, H. R., \& Sing, D. K. 2015, A\&A, 573, A122

Wargelin, B. J., Saar, S. H., Pojmański, G., Drake, J. J., \& Kashyap, V. L. 2017 MNRAS, 464, 3281

Weber, M., Granzer, T., \& Strassmeier, K. G. 2012, in SPIE Conf. Ser., 8451

Youngblood, A., France, K., Parke Loyd, R. O., et al. 2017, ApJ, 843, 31 